\title{
Image formation and optical transfer function in a course of Fourier optics
}

\section{Maria Yzuel}

Maria Josefa Yzuel, "Image formation and optical transfer function in a course of Fourier optics," Proc. SPIE 3831, Sixth International Conference on Education and Training in Optics and Photonics, (16 June 2000); doi: $10.1117 / 12.388720$

Event: Education and Training in Optics and Photonics (ETOP'99), 1999, Cancun, Mexico 


\title{
Image formation and optical transfer function in a course of Fourier Optics.
}

\author{
María J. Yzuel \\ Departamento de Física, Grupo de Óptica, Universidad Autónoma de Barcelona, 08193 Bellaterra \\ SPAIN, e-mail YZUEL@cc.uab.es
}

\begin{abstract}
A discussion on the interest of the study of diffractional image formation theory in a course of Fourier Optics is carried out. We develope a general description of the partially coherent illumination case. Images of extended objects like the line and periodical structures are given for different values of the coherence parameter. The apparent transfer function is introduced as a quality criterium. For the incoherent illumination this parameter is compared with the optical transfer function.
\end{abstract}

Keywords: Partially coherent illumination, Diffractional image formation, Apparent Transfer Function

\section{INTRODUCTION}

The rather recent developments in Optics have increased the number of people working in this field. A course of Fourier Optics is introduced in many universities in the degrees for physicists and engineers. In such a course the image formation fundamentals can be tought in the context of linear systems ${ }^{1,2}$. The impulse response is identified with the point spread function. The frequency analysis leads to the optical transfer function. The wave aberration ${ }^{3}$ can be introduced in this approach and it allows the study of the effect of the residual aberrations in the image.

It is interesting to teach the student that the image formation of extended objects and the analysis of the frequency transmission depend on the kind of illumination. A complete study must consider the incoherent, coherent and partially coherent illumination. A discussion on the linearity or non-linearity of the system response in each case is interesting.

The words Fourier Optics are identified with a course in Information Processing, but in most of the text books in this subject one can find a chapter or a section of a chapter on the theory of imaging systems. For extended objects with incoherent illumination the similarity with linear systems, and the frequency analysis in the transmission from the object to the image are described in terms of the Optical Transfer Function. Baker ${ }^{4}$ has edited a selection of papers on the foundation and theory of the Optical Transfer Function as well as the measurements of this function in optical systems.

Actually, in a course of Fourier Optics the students have the tools to be trained on the image formation theory, taking into account diffraction and the pupil function with wave aberration. Quality criteria, like Strehl ratio, resolving power and Optical Transfer Function can also be introduced.

A background of diffraction theory is necessary before the study of image formation. Only in case of a geometrical approach it is possible to give the Point Spread Function as a distribution of dots that consists of the cuts of the rays with the image plane.

In many books ${ }^{1,5}$ the point spread function and the image of extended objects is treated. They generaly deal with incoherent or coherent illumination.

In this paper a theory of image formation in partially coherent illumination, that is the general case, is described. Some results for extended objects like the line or periodical structures are given to show the influence of the coherence degree. This approach could be more difficult than the expected one in a course of Fourier Optics, but it is a further step to complete the student training that could be introduced in a seminar or in a Ph. D. Course. 


\section{IMAGE FORMATION WITH PARTIALLY COHERENT ILLUMINATION}

\subsection{The calculation of the 3D line spread function}

All optical systems, which image small structures up to the size of the wavelenght show partially coherence. That means the illumination used is partially coherent. This property can be described by the mutual coherence function $\Gamma\left(\overrightarrow{\mathrm{x}}_{01}, \overrightarrow{\mathrm{x}}_{02}\right)^{6}$. We made a development of the formulation of the image formation for partially coherent illumination ${ }^{7,8}$.

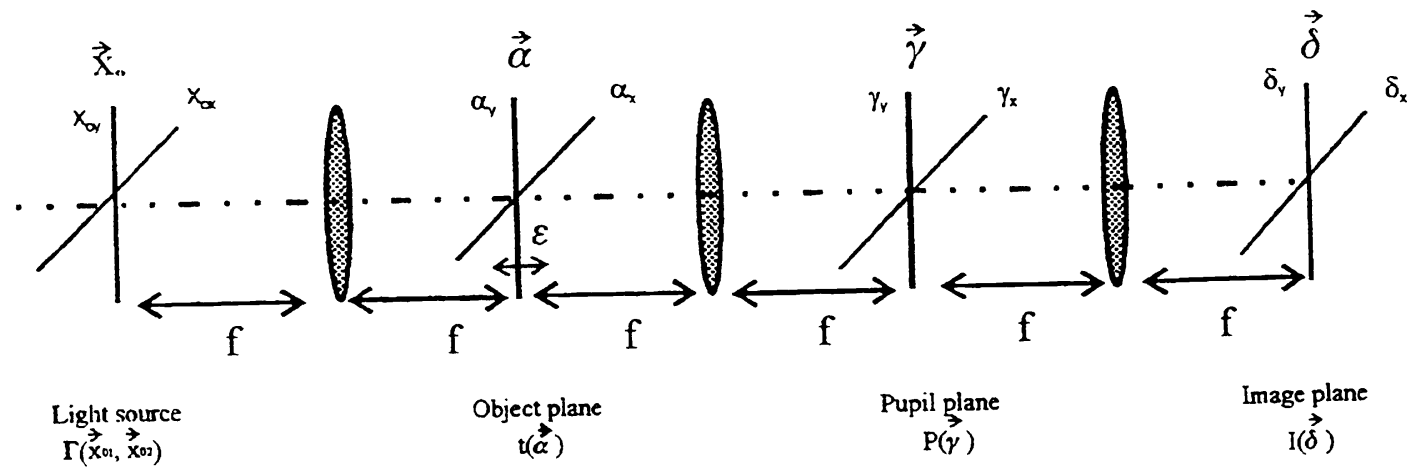

Fig. 1. Model of an optical imaging system

Applying the propagation law of the mutual coherence function in the optical system given by fig. 1, the image intensity can be calculated as:

$$
\begin{aligned}
\mathrm{I}\left(\vec{\delta}, \mathrm{W}_{20}\right) & =\int \cdots(12) \int \Gamma\left(\overrightarrow{\mathbf{x}}_{01}, \overrightarrow{\mathbf{x}}_{02}\right) \mathrm{t}\left(\vec{\alpha}_{1}\right) \mathrm{t}^{*}\left(\vec{\alpha}_{2}\right) \mathrm{P}\left(\vec{\gamma}_{1}\right) \mathrm{P}^{*}\left(\vec{\gamma}_{2}\right) \exp \left[2 \pi \mathrm{iW} \mathrm{W}_{20}\left(\vec{\gamma}_{1}^{2}-\vec{\gamma}_{2}^{2}\right)\right] \\
& \cdot \exp \left[\mathrm{i}\left(\vec{\alpha}_{1} \overrightarrow{\mathbf{x}}_{01}-\vec{\alpha}_{2} \overrightarrow{\mathbf{x}}_{02}\right)\right] \exp \left[\mathrm{i}\left(\vec{\alpha}_{1} \vec{\gamma}_{1}-\vec{\alpha}_{2} \vec{\gamma}_{2}\right)\right] \exp \left[\mathrm{i} \vec{\delta}\left(\vec{\gamma}_{1}-\vec{\gamma}_{2}\right)\right] \mathrm{d} \overrightarrow{\mathrm{x}}_{01} \ldots \mathrm{d} \vec{\gamma}_{2}
\end{aligned}
$$

The coordinates used in eq. (1) correspond to the coordinates used in fig. 1. They can be considered as Hopkins canonical coordinates $^{9}$ related to the wavelenght $\lambda$, to the numerical aperture of the optical system (NA) and to the metrical coordiantes by:

$$
\vec{\alpha}=\frac{2 \pi}{\lambda} \mathrm{NA} \vec{\alpha}_{m} ; \quad \vec{\delta}=\frac{2 \pi}{\lambda} \mathrm{NA}_{\mathrm{m}}
$$

The vector symbol is to indicate that we have to consider the two dimensions

$$
\vec{\alpha}=\left(\alpha_{x}, \alpha_{y}\right) ; \ldots ; \vec{\delta}=\left(\delta_{x}, \delta_{y}\right)
$$

The transmission of the object is described by the function $t(\vec{\alpha})$. The influence of the axial shift of the object plane $(\varepsilon)$, to describe the $3 \mathrm{D}$ response behaviour is also given by a complex function in the pupil plane. It is given by $\exp \left(\mathrm{i} 2 \pi \mathrm{W}_{20} \gamma^{2}\right)$ where $W_{20}$ is connected with the shift of the object plane $\varepsilon$ through:

$$
\mathrm{W}_{20}=\frac{1}{2} \frac{\varepsilon \mathrm{NA}^{2}}{\lambda}
$$


Possible optical filters or wave aberration are introduced in the pupil funciton $\mathrm{P}(\gamma)$. The Hopkins canonical coordinates are normalized so that the maximum radius in the pupil is equal to one. If an apodizing filter is introduced in the diffraction limited system, the pupil function $\mathrm{P}(\gamma)$ is equal to the filter transmission and it is given by $\tau(\gamma)$ inside the pupil and zero outside. The integration over the light source in eq. (1) leads to a mutual coherence function $\Gamma\left(\alpha_{1}, \alpha_{2}\right)$ in the object plane. Then the image intensity is described by:

$$
\begin{aligned}
\mathrm{I}\left(\delta, \mathrm{W}_{20}\right)= & \int \cdots_{(8)} \int \Gamma\left(\mathrm{x}_{01}, \mathrm{x}_{02}\right) \mathrm{t}\left(\alpha_{1}\right) \mathrm{t}^{*}\left(\alpha_{2}\right) \mathrm{P}\left(\gamma_{1}\right) \mathrm{P}^{*}\left(\gamma_{2}\right) \exp \left[2 \pi \mathrm{iW} \mathrm{W}_{20}\left(\gamma_{1}^{2}-\gamma_{2}^{2}\right)\right] \\
& \cdot \exp \left[\mathrm{i}\left(\alpha_{1} \gamma_{1}-\alpha_{2} \gamma_{2}\right)\right] \exp \left[\mathrm{i} \delta\left(\gamma_{1}-\gamma_{2}\right)\right] \mathrm{d} x_{1} \ldots \mathrm{d} \gamma_{2}
\end{aligned}
$$

A further interpretation of eq. (3) is not possible, i.e. one cannot define a transfer function of the optical system without special assumptions about the object or the light source.

First of all let us suppose that there is coherent illumination in the object plane $\Gamma\left(\alpha_{1}, \alpha_{2}\right)=1$. From eq. (3) one gets:

$$
\left.\mathrm{I}\left(\delta_{\mathrm{x}}, \delta_{\mathrm{y}}, \mathrm{W}_{20}\right)=\mid \iint \widetilde{\mathrm{t}}\left(\gamma_{\mathrm{x}}, \gamma_{\mathrm{y}}\right) \mathrm{P}\left(\gamma_{\mathrm{x}}, \gamma_{\mathrm{y}}\right) \exp \left[2 \pi \mathrm{iW} \mathrm{W}_{20}\left(\gamma_{\mathrm{x}}^{2}+\gamma_{\mathrm{y}}^{2}\right)\right] \exp \left[\mathrm{i} \delta_{\mathrm{x}} \gamma_{\mathrm{x}}+\delta_{\mathrm{y}} \gamma_{\mathrm{y}}\right)\right]\left.\mathrm{d} \gamma_{\mathrm{x}} \mathrm{d} \gamma_{\mathrm{y}}\right|^{2}
$$

where $\tilde{\mathrm{t}}$ means the Fourier transform of $\mathrm{t}$.

If the object is a line or a narrow slit parallel to the y-axis.

$$
\mathbf{t}\left(\alpha_{x}, \alpha_{y}\right)=\delta\left(\alpha_{x}\right)
$$

then

$$
\mathrm{I}\left(\delta_{\mathrm{x}}, \mathrm{W}_{20}\right)=\left|\iint \mathrm{P}\left(\gamma_{\mathrm{x}}, 0\right) \exp \left[2 \pi \mathrm{i} \mathrm{W}_{20} \gamma_{\mathrm{x}}^{2}\right] \exp \left[\mathrm{i} \delta_{\mathrm{x}} \gamma_{\mathrm{x}}\right] \mathrm{d} \gamma_{\mathrm{x}}\right|^{2}
$$

That is, for coherent illumination, the $3 \mathrm{D}$ line response is described by the transmission behaviour along one axis ${ }^{10}$.

The interpretation of eq. (1) is also possible with the assumption of the effective incolerent light source introduced by Hopkins $^{11}$.

$$
\Gamma\left(x_{01}, x_{02}\right)=I_{0} \cdot \delta\left(x_{01}-x_{02}\right)
$$

then

$$
\mathrm{I}\left(\delta_{\mathrm{x}}, \mathrm{W}_{20}\right)=\int \mathrm{S}\left(\mathrm{x}_{0}\right) \mathrm{d} \mathrm{x}_{0}\left|\int \tilde{\mathrm{t}}\left(\gamma+\mathrm{x}_{0}\right) \exp \left[2 \pi \mathrm{i} \mathrm{W}_{20} \gamma^{2}\right] \mathrm{P}(\gamma) \exp [\mathrm{i} \delta \gamma] \mathrm{d} \gamma\right|^{2}
$$

where $S\left(x_{0}\right)$ is the effective incoherent light source and describes the emission of the effective light source e.g. circular light source of radius $\mathrm{S}$. By using the reduced coordinates its size is given by the ratio of the numerical aperture of the condenser to the numerical aperture of the objective and it is called "coherence parameter".

If we consider a line object given by eq. (5) and a circular incoherent radiating light source with radius $S$, it is possible to calculate the line response function for radial symmetric pupil transmissions by the following expression:

$$
\mathrm{I}\left(\delta_{\mathrm{x}}, \mathrm{W}_{20}\right)=\left.\int_{-s}^{\mathrm{s}} 2 \sqrt{\mathrm{S}^{2}-\mathrm{x}_{0 \mathrm{y}}^{2}} \int_{-\sqrt{1-x_{0 y}^{2}}}^{\sqrt{1-x_{0 y}^{2}}} \mathrm{P}\left(\sqrt{\gamma_{\mathrm{x}}^{2}+\mathrm{x}_{0 \mathrm{y}}^{2}}\right) \cdot \exp \left[2 \pi \mathrm{i} \mathrm{W}_{20} \gamma_{\mathrm{x}}^{2}\right] \exp \left[\mathrm{i} \delta_{\mathrm{x}} \gamma_{\mathrm{x}}\right] \mathrm{d} \gamma_{\mathrm{x}}\right|^{2} \mathrm{~d} \mathrm{x}_{0 \mathrm{y}}
$$

The coherence parameter for circular effective source will be given by its radius (S). 
For the circular source, $S=0$ describes the case of coherent illumination and $S=\infty$ describes the incolherent light source. For the calculation of the transmission behaviour of the optical system usually a small value of $S$ is used to describe the quasicoherent illumination, e.g. $S=0.2$. Partial coherence is characterized by a coherence factor $S$ between 0 and 1 . The incoherent case is characterized by a number for $\mathrm{S}$ higher than 1 .

For a rectangular light source $\left(-S_{x} ;-S_{y} ; S_{x} ; S_{y}\right)$ the intensity in the line response is given by

$$
I\left(\delta_{x}, W_{20}\right)=\left.\int_{-s_{y}}^{s_{y}} 2 S_{x} \int_{-\sqrt{1-x_{0 y}^{2}}}^{\sqrt{1-x_{0 y}^{2}}} P\left(\sqrt{\gamma_{x}^{2}+x_{0 y}^{2}}\right) \cdot \exp \left[2 \pi i W_{20} \gamma_{x}^{2}\right] \exp \left[i \delta_{x} \gamma_{x}\right] d \gamma_{x}\right|^{2} d x_{0 y}
$$

In this case the coherence parameter of the optical system is only influenced by the extension of the light sourece in $\mathrm{x}_{0 y}$ direction. The contribution of the source length along the $x_{0 x}$ direction is only a contribution to the amount of energy in the image.

The calculation of the line spread function (LSF) for incoherent illumination is also possible in the way proposed by Williams and Becklund ${ }^{12}$.

The starting point of Williams and Becklund is the point spread function (PSF) in the best image plane. This function is equal to the Fouricr Bessel Transform for the radial symmetric case

$$
\mathrm{PSF}=\left|\int_{0}^{1} \mathrm{P}(\rho) \cdot \rho \cdot \mathrm{J}_{0}(\mathrm{r} \rho) \mathrm{d} \rho\right|^{2}
$$

The line spread function (LSF) is then given by the convolution of this PSF with the intensity of the line in y-direction.

Eq. (10) can be obtainded in defocused planes by introducing a modified pupil P' $(\rho)$ given by

$$
P^{\prime}(\rho)=P(\rho) \cdot \exp \left[2 \pi i W_{20} \rho^{2}\right]
$$

instead of $P(\rho)$ in (9).

So it is possible to calculate the $3 \mathrm{D}$ line response function by the convolution of the line intensity with the point spread function for each $\mathrm{W}_{20}$

$$
\mathrm{I}\left(\delta_{x}, \mathrm{~W}_{20}\right)=\int_{-\infty}^{\infty} \operatorname{PSF}\left(\delta_{x}, \delta_{y}, \mathrm{~W}_{20}\right) \mathrm{d} \delta_{y}
$$

With eq. (8) and eq. (12) we have two different possibilities to calculate the 3D LSF. Both of them were programmed on the computer and yield identical results in the incoherent case. However the eq. (12) needs longer computing time and in contrast to eq. (8), is for incoherent illumination only.

The 3D coherent line response and the line spread function (LSF) are drawn in fig. 2a and $b$ for the diffraction limited pupil. The 3D plot on the top shows more oscillations with clear minima along the optical axis and in the best image plane $\left(W_{20}=0\right)$ for the case of coherent illumination. These side lobes are strongly reduced for incoherent illumination. Especially in the best image plane one can see a smoothed behaviour. Cuts in different focus planes are drawn in fig. $2 c$ and $d$. In the best image plane $\left(\mathrm{W}_{20}=0\right)$ one can see the well-known line responses e.g. from ${ }^{10,11}$. Fig. $2 \mathrm{c}$ and $2 \mathrm{~d}$ shows that the influence of defocus is stronger in the case of coherent illumination. 

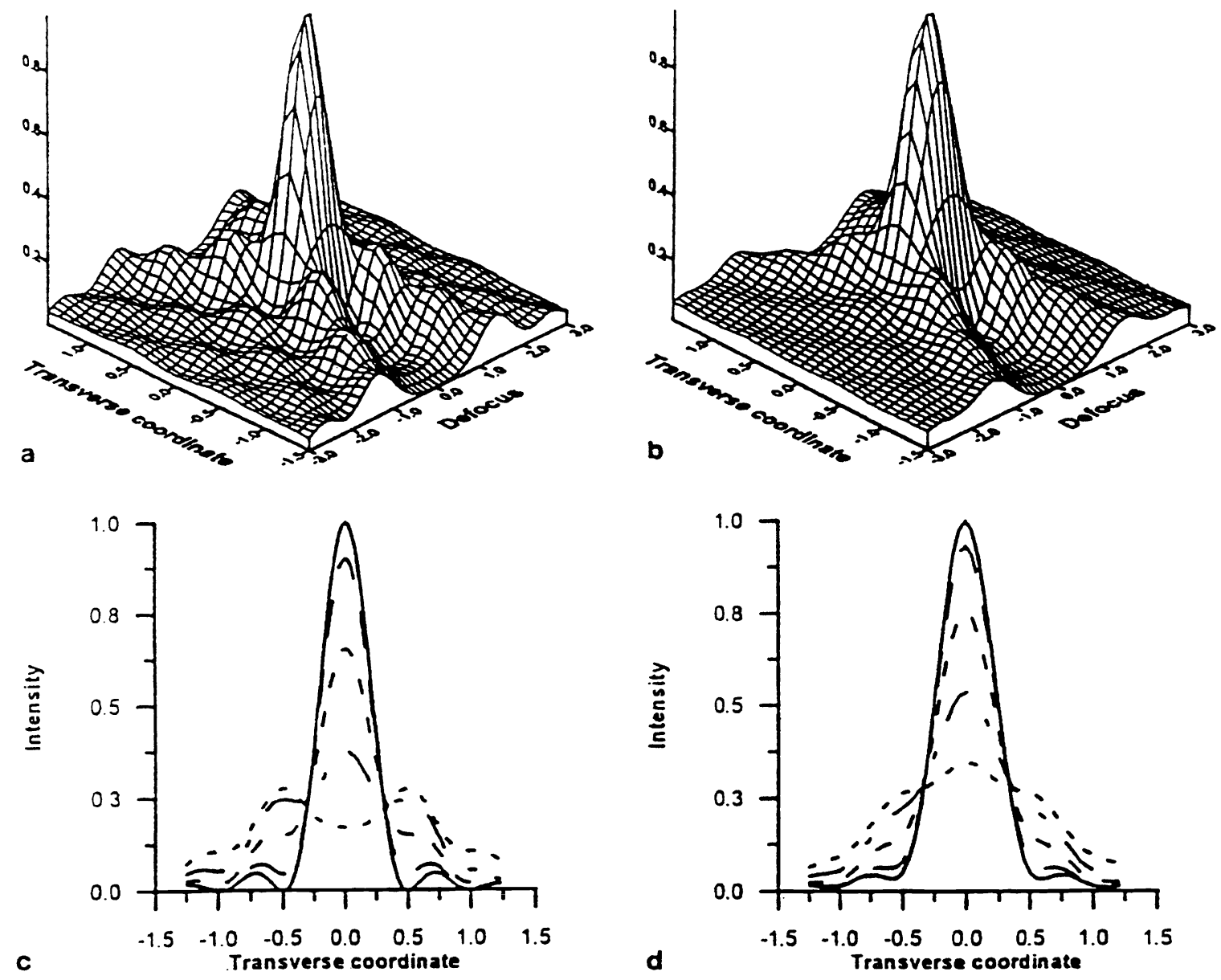

Fig 2. a) Comparison of the 3D coherent line response and b) the Line Spread Function LSF for the diffraction limited optical system. c) transversal sections of fig. $2 \mathrm{a}$ in defocused planes $\mathrm{W} 20=0$ (solid), W20 $=0.17$ (dashed), W20 $=0.34$ (small dashed), W20 $=0.51$ (dashed dotted), $\mathrm{W} 20=0.68$ (dotted), d) transversal sections of fig. $2 \mathrm{~b}$ in the same defocused planes.

\subsection{Periodical structures. Apparent transfer function}

If we consider a sinusoidal transmission object given by

$$
t\left(\alpha_{x}\right)=a+b \cdot \cos \left(2 \pi \mu \alpha_{x}\right)
$$

where $a$ and $b>0$ and $a \geq b, \mu$ is the spatial frecuency. The Fourier Transform of the object becomes:

$$
\tilde{\mathbf{t}}\left(\gamma_{\mathrm{x}}+\mathrm{x}_{0 \mathrm{x}}\right)=\mathbf{a} \delta\left(\gamma_{\mathrm{x}}+\mathrm{x}_{0 \mathrm{x}}\right)+\frac{1}{2} \mathbf{b}\left[\delta\left(\gamma_{\mathrm{x}}+\mathrm{x}_{0 \mathrm{x}}+\mu\right)+\delta\left(\gamma_{\mathrm{x}}+\mathrm{x}_{0 \mathrm{x}}-\mu\right)\right] .
$$


We obtain the intensity distribution in the image of a sinusoidal transmission object given by

$$
\begin{aligned}
I\left(\delta_{x}\right) & =\iint S\left(x_{0 x}, x_{0 y}\right) d x_{0 x} d x_{0 y} \mid \int \operatorname{aP}\left[\left(x_{0 x}^{2}+x_{0 y}^{2}\right)^{1 / 2}\right] \\
& \left.+\frac{1}{2} b \cdot \exp \left[i 2 \pi W_{20} \mu^{2}\right] P P\left[\left(x_{0 x}-\mu\right)^{2}+x_{0 y}^{2}\right]^{1 / 2}\right] \exp \left[i 2 \pi\left(-W_{20} \mu x_{0 x}+\delta_{x} \mu\right)\right] \\
& \left.+P\left[\left[\left(x_{0 x}+\mu\right)^{2}+x_{0 y}^{2}\right]^{/ 2}\right] \exp \left[i 2 \pi\left(-W_{20} \mu x_{0 x}-\delta_{x} \mu\right)\right]\right\}^{2}
\end{aligned}
$$

To measure the imaging behaviour of the optical system for partially coherent illumination, the Apparent Transfer Function (ATF) can be introduced ${ }^{13,14}$. The ATF is defined by the ratio of the contrast in the image and the contrast in the object when the system makes the image of a cosine amplitude test object. The object is given by eq. (13). The contrast in the intensity distribution on the object is

$$
\theta_{0}=\frac{I_{\max }-I_{\min }}{I_{\max }+t_{\min }}=\frac{2 a b}{a^{2}+b^{2}}
$$

and it does not depend on the spatial frequency $\mu$. The intensity distribution in the image is given by eq. (15). We evaluate $I\left(\delta_{x}\right)$ for $\delta_{x}$ values in a spatial period and the maximum $\left(I_{\max }\right)$ and minimum $\left(I_{\min }\right)$ values are obtained. The contrast in the image is given by

$$
\theta_{i}=\frac{I_{\max }-I_{\min }}{I_{\max }++_{\min }}
$$

where $\theta_{\mathrm{i}}$ depends not only on the frequency and the defocus but also on the degree of coherence of the illumination. This parameter is related with the size $S$ of the effective source. The ATF is defined by

$$
\operatorname{ATF}(\mu)=\frac{\theta_{i}(\mu)}{\theta_{0}}
$$

This function is evaluated for different transmissions pupils and different degrees of coherence.

For incoherent illumination, the OTF is a very well known parameter used to evaluate the quality of an optical system. Note that the ATF evaluated in the incoherent cases does not coincide with the OTF, because the ATF is obtained as the contrast of the image of a sinusoidal amplitude transmission object. In intensity transmission, this object also presents a double harmonic. In effect, if the object has a sinusoidal transmission in amplitude [given by Eq. (13)], the intensity distribution is

$$
\begin{aligned}
T\left(\alpha_{x}\right) & =\left|a+b \cos \left(2 \pi \mu \alpha_{x}\right)\right|^{2} \\
& =a^{2}+\frac{b^{2}}{2}+2 b a \cdot \cos \left(2 \pi \mu \alpha_{x}\right)+\frac{b^{2}}{2} \cos \left(2 \pi\left(2 \mu \alpha_{x}\right)\right)
\end{aligned}
$$

In the image of this object, for the incoherent illumination, the distribution of intensity is

$$
\mathrm{I}\left(\alpha_{\mathrm{x}}\right)=\left(\mathrm{a}^{2}+\frac{\mathrm{b}^{2}}{2}\right) \mathrm{OTF}(0)+2 \mathrm{ba} \cdot \mathrm{OTF}(\mu) \cdot \cos \left(2 \pi \mu \alpha_{\mathrm{x}}\right)+\frac{\mathrm{b}^{2}}{2} \mathrm{OTF}(2 \mu) \cdot \cos \left(2 \pi\left(2 \mu \alpha_{\mathrm{x}}\right)\right),
$$

where $\operatorname{OTF}(\mu)$ is the OTF for the frequency $\mu$. We normalize $\operatorname{OTF}(0)=1$. The contrast of the intensity distribution given by Eq.(20) depends on the values of the frequencies $\mu$ and $2 \mu$.

Fig. 3 shows the apparent transfer function for the diffraction limited system, for two values of the coherence $S=0.5$ that represent partially coherence illumination and $S=4$ that gives incoherent illumination. 


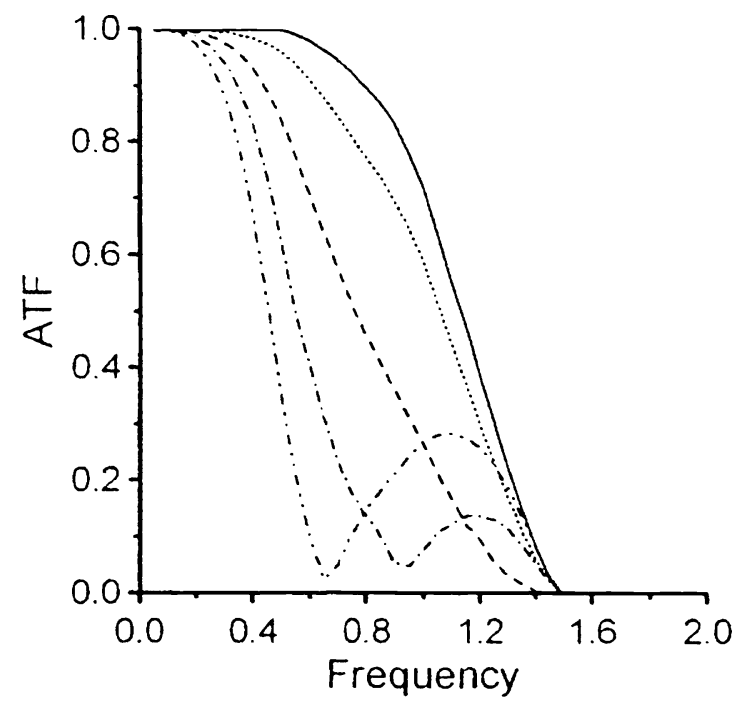

(a)

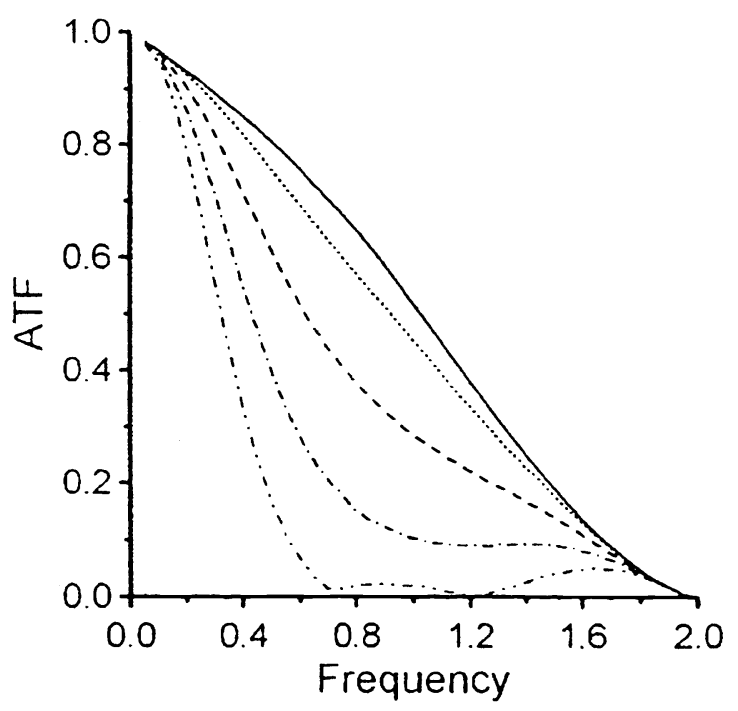

(b)

Fig 3. ATF given by the diffraction limited system at various defocused planes $\left(W_{20}=0\right.$, solid line; $W_{20}=0.17$, dotted line; $W_{20}=0.34$, dashed line; $W_{20}=0.51$, dash-dotted line; $\mathrm{W}_{20}=0.68$, dash-double-dotted line) and two values of the coherence parameter: (a) $\mathrm{S}=0.5$ and (b) $\mathrm{S}=4$.

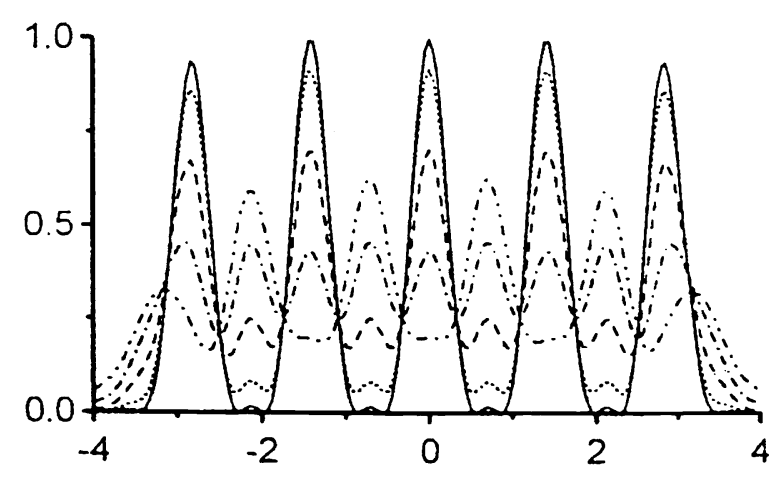

(a)

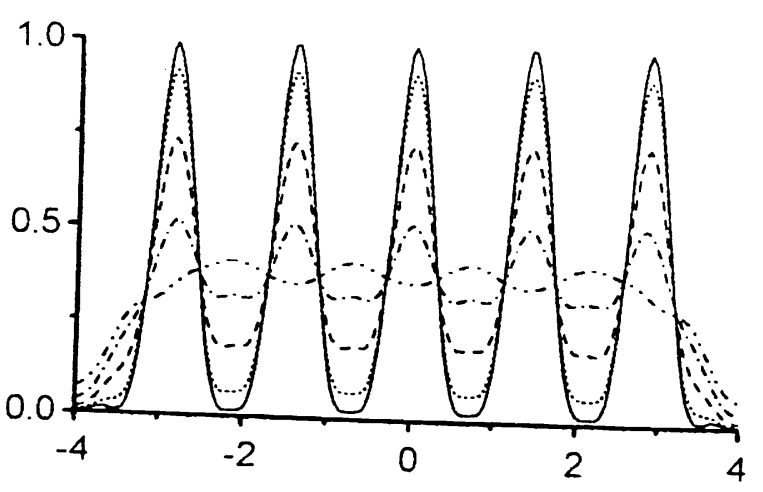

(b)

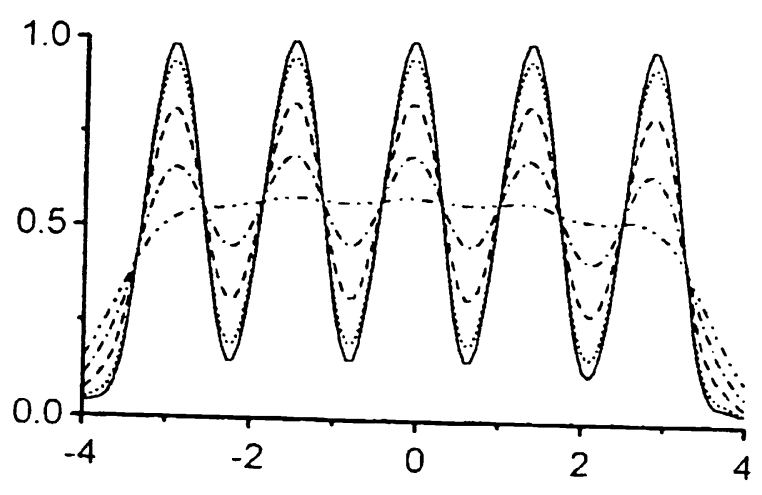

(c)

Fig 4. Images of the five-bar test given the diffraction limited system at various defocused planes $\left(\mathrm{W}_{20}=0\right.$, solid line; $\mathrm{W}_{20}=0.17$, dotted line; $\mathrm{W}_{20}=0.34$, dashed line; $\mathrm{W}_{20}=0.51$, dash-dotted line; $W_{20}=0.68$, dash-double-dotted line) and with different values of the coherence parameter: (a) $\mathrm{S}=0.2$, (b) $\mathrm{S}=0.5$, and (c) $\mathrm{S}=\infty$. 
We have also computed the image of a five-bar object. The dimensions of the bars are $0.52 \mu \mathrm{m}$ and the period is $1.04 \mu \mathrm{m}$. The frequency of this five-bar object in the units used in Eq.(2) is 0.702 for NA=0.5 and $\lambda=0.365 \mu \mathrm{m}$.

Fig. 4 shows the images of the five-bar test given by a system without aberration and with different coherence parameter values [different sizes of the effective light source]: Fig. $4 a \mathrm{a}=0.2$, Fig. $4 \mathrm{~b} S=0.5$, Fig. $4 \mathrm{c} S=\infty$. Each figure shows the images of the five bars on different defocused planes: $W_{20}=0, W_{20}=0.17, W_{20}=0.34, W_{20}=0.51$, and $W_{20}=0.68$. A common behaviour shown in all the figures is that the contrast of the bars decreases as the defocusing increases

Fig. 4a corresponds to a partially coherent illumination $(S=0.2)$ not very different from the pure coherent case ( $S=0$ ), we call it quasi-coherent illumination. For $\mathrm{W}_{20}=0.51$, a double frequency is produced, and for $\mathrm{W}_{20}=0.68$, a contrast inversion appears.

Fig. $4 \mathrm{~b}$ corresponds to the case of partially coherent illumination $(S=0.5)$, which is representative of the type of illumination that appears for example in microlithography, and shows that the contrast of the bars decreases with defocus, but at the plane $\mathrm{W}_{20}=0.51$, the five bars are still recognized [the double frequency does not appear]. This means that with $S=0.5$ the depth of focus is higher. The contrast at the best image plane is lower than for smaller values of S. A contrast inversion appears at the plane $\mathrm{W}_{20}=0.68$, but the contrast here is very low.

Fig. $4 c$ corresponds to the pure incoherent illumination $(S=\infty)$. It also shows that the contrast of the bars decreases with defocus, but neither a double frequency nor a contrast inversion appears. The bar images are almost a constant for $\mathrm{W}_{20}=0.68$. The depth of focus is slightly higher than in the previous cases, but the contrast at the best image plane is the lowest.

These results can be understood with the Apparent Transfer Function. Fig. 3a shows that the value of the ATF at the frequency 0.7 that corresponds to the frequency of the five-bar test, decreases with defocus at the planes $W_{20}=0,0.17,0.34$, and 0.51 . Nevertheless, at the plane $\mathrm{W}_{20}=0.68$ the value of the ATF, although small, increases from an inflexion point at a frequency of $\sim 0.6$. Since the ATF is calculated in modulus, this part of the curve represents a contrast inversion, a fact that is supported by the contrast inversion observed in that plane in Fig. $4 \mathrm{~b}$.

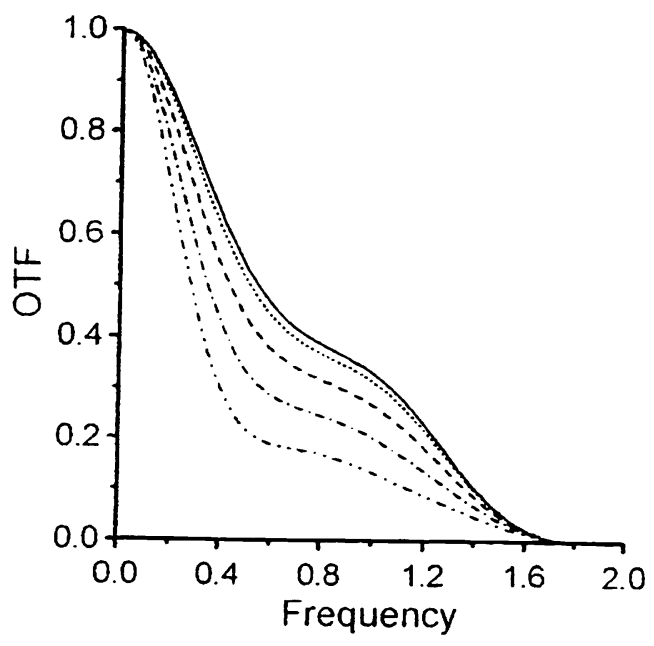

Fig 5. OTF given by the diffraction limited system at various defocused planes $\left(W_{20}=0\right.$, solid line; $W_{20}=0.17$, dotted line; $W_{20}=0.34$, dashed line; $\mathrm{W}_{20}=0.51$, dash-dotted line; $\mathrm{W}_{20}=0.68$, dash-double-dotted line). 
Fig. $3 \mathrm{~b}$ shows the ATF with incoherent illumination. It shows that the contrast is reduced in comparison to the partially coherent case, which confirms the observations made in the five-bar test. Nevertheless, the limit frequency was 1.5 with $\mathrm{S}=0.5$, and with incoherent illumination it is 2 , which means that higher frequencies can be transmitted when the coherence parameter increases.

We now analyze the relation between the ATF for incoherent illumination and the OTF. The image of the sinusoidal object is given by Eq. (20). If the OTF fulfills the following conditions: $\operatorname{OTF}(\mu)>0, \operatorname{OTF}(2 \mu)>0$, and $\operatorname{OTF}(\mu) \geq \operatorname{OTF}(\mu)$, then the contrast in the incoherent image of the sinusoidal object is

$$
\theta_{i}=\frac{2 \mathrm{ba}|\operatorname{OTF}(\mu)|}{\mathbf{a}^{2}+\left(\mathbf{b}^{2} / 2\right)+\left(\mathbf{b}^{2} / 2\right)|\operatorname{OTF}(2 \mu)|}
$$

If we analyze $a=0.5$ and $b=0.5$, then the contrast in the object given by Eq.(16) is equal to 1 , and the ATF becomes

$$
\operatorname{ATF}(\mu)=\frac{4|\operatorname{OTF}(\mu)|}{3+|\operatorname{OTF}(2 \mu)|} \text {. }
$$

In the examples we have studied, the conditions mentioned are fulfilled except for the plane $W_{20}=0.68$. Thus, the relation between the ATF and the OTF is given by Eq. $(22)$. When the $\operatorname{OTF}(2 \mu)=0$, the $\operatorname{ATF}(\mu)=(4 / 3) \operatorname{OTF}(\mu)$. In general this situation happens for $\mu \geq 1$. As the $\operatorname{OTF}(2 \mu) \leq 1$, the denominator of $\operatorname{Eq}(22)$ is always less than 4 and in consequence, $\operatorname{ATF}(\mu)$ is larger than the $\operatorname{OTF}(\mu)$. In fig. 5 we show the OTF for the diffraction limited case. By comparing fig. 5 with fig. $3 \mathrm{~b}$ we can observe the difference between the ATF and the OTF.

\section{SUMMARY}

The interest of introducing the theory of diffractional image formation in a course of Fourier Optics is discussed. The students have the tools of Fourier analysis and linear systems behaviour to understand the fundamentals of image formation in incoherent and coherent illumination. These concepts are already developed in many text books. Here, a general approach for the image formation in partially coherent illumination is described. The student can learn from the results obtainded with extended objects. The line response and the image of periodical structures are obtainded for different values of the coherence parameter. The apparent transfer function is analyzed. Finally, the comparison between the ATF for incoherent illumination and the Optical Transfer Function is analyzed.

\section{ACKNOWLEDGEMENTS}

I would like to thank Drs. R. Hild, J.C. Escalera and J. Campos, for the collaboration in the research in image formation in partially coherent illumination. I also thank Dr. I. Moreno and Mr. J. Nicolas for their assistance in preparing this manuscript.

\section{REFERENCES}

1. J.W. Goodman, Introduction to Fourier Optics, McGraw-Hill, 1968, $2^{\text {nd }}$ edition 1997.

2. W.T. Cathey, Optical Information Processing and Holography, Krieger Publishers, Melbourne (USA).

3. H.H, Hopkins, Wave Theory of Aberrations, Clarendon Press, Oxford 1950.

4. L. Baker, ed. Selected Papers on Optical Transfer Function, Voll. Foundation and Theory Vol.2: Measurements, SPIE Milestone Series, SPIE Optical Engineering Press, Vol.MS59 and MS60, Bellingham 1992.

5. A. Maréchal, M. Françon. Diffraction, structure des images, Éd. de la revue d'optique théorique et instrumentale, Paris, 1960. 
6. M. Born, E.Wolf, Principles in Optics. Chapter X. Pergamon Press. $3^{\text {rd }}$. Edition, London, 1965

7. R.Hild, M.J. Yzuel, J.C. Escalera, "Influence of optical filtering and coherence parameter on the 3-D line spread function in photolithografic imaging", Optik 102, pp. 159-167, 1996

8. R.Hild, M.J. Yzuel, J.C. Escalera, J. Campos, "Influence of nonuniform pupils in imaging periodical structures by photolithographic systems", Opt. Eng. 37, V.4, pp. 1353-1363, 1998

9. H.H. Hopkins, "Canonical and Real-Space Coordinates used in the Theory of Image Formation". Applied Optics and Optical Engineering. In R.R. Shannon, J.C.Wyant (Eds). pp. 307-369. Academic Press, Orlando, FL 1983.

10. D. Gaskill Linear Systems, Fourier Transforms, and Optics. John Wiley \& Sons, New York 1978.

11. H.H. Hopkins, "Applications of the coherence theory in microscopy and interferometry". J. Opt. Soc. Am. 47, pp. 508526,1957

12. C.S. Williams, O.A. Becklund, Optical Transfer Function, pp 57, John Wiley \& Sons, New York, 1989.

13. S. Kessler, J.Hebenstreit and G. Nitzsche, "Spatial filtering with compelementary source pupil masks", J. Mod. Opt. 36, 119-132, 1989.

14. F.T.S. Yu, White Light Optical Signal Processing, John Wiley \& Sons, New York, 1985. 